\title{
Control of On/Off Glomerular Signaling by a Local GABAergic Microcircuit in the Olfactory Bulb
}

\author{
David H. Gire ${ }^{1}$ and Nathan E. Schoppa ${ }^{2}$ \\ ${ }^{1}$ Neuroscience Program and 2Department of Physiology and Biophysics, University of Colorado Denver, Anschutz Medical Campus, Aurora, \\ Colorado 80045
}

\begin{abstract}
Odors are coded at the input level of the olfactory bulb by a spatial map of activated glomeruli, reflecting different odorant receptors (ORs) stimulated in the nose. Here we examined the function of local synaptic processing within glomeruli in transforming these input patterns into an output for the bulb, using patch-clamp recordings and calcium imaging in rat bulb slices. Two types of transformations were observed at glomeruli, the first of which produced a bimodal, "on/off" glomerular signal that varied probabilistically depending on olfactory receptor neuron (ORN) input levels. The bimodal response behavior was seen in glomerular synaptic responses, as well as in action potential ("spike") firing, wherein all mitral cells affiliated with a glomerulus either engaged in prolonged spike bursts or did not spike at all. In addition, evidence was obtained that GABAergic periglomerular (PG) cells that surround a glomerulus can prevent activation of a glomerulus through inhibitory inputs targeted onto excitatory external tufted cells. The path of PG cell activation appeared to be confined to one glomerulus, such that ORNs at one glomerulus initiated inhibition of the same glomerulus. The observed glomerular "self-inhibition" provides a mechanism of filtering odor signals that would be an alternative to commonly proposed mechanisms of lateral inhibition between OR-specific glomeruli. In this case, selective suppression of weak odor signals could be achieved based on the difference in the input resistance of PG cells versus excitatory neurons at a glomerulus.
\end{abstract}

\section{Introduction}

Odors elicit a spatial map of activated glomeruli in the olfactory bulb, with each glomerulus reflecting one odorant receptor (OR) stimulated in the nose (Bozza and Mombaerts, 2001). How this input is transformed by the bulb circuitry into an output, however, remains unresolved. One commonly proposed function of the bulb is that it acts as a signal filter, whereby strongly activated OR-specific glomeruli suppress weakly activated glomeruli through lateral inhibition (Mori et al., 1999; Urban, 2002; Aungst et al., 2003; Shepherd et al., 2007; Arevian et al., 2008). Such a function, analogous to signal filtering mechanisms in other sensory systems (Kuffler, 1953; Yang et al., 1992), could be important for olfactory contrast enhancement (Yokoi et al., 1995). However, a recent study (Soucy et al., 2009) suggested that bulb glomeruli are not well ordered by functional type in the manner of neurons in other sensory systems, in which case lateral inhibition may be a less effective signal filter.

An alternate mechanism for odor signal filtering that would be unaffected by glomerular order involves local synaptic processing within a single glomerulus. The key players in such a mechanism could be GABAergic periglomerular (PG) cells (Panzanelli et al.,

Received May 20, 2009; revised Sept. 14, 2009; accepted Sept. 16, 2009.

This work was supported by National Institutes of Health Grants 2T32 NS0077083 and F31 DC009118 (D.H.G) and R01 DC006640 (N.E.S). We thank Drs. Diego Restrepo and Sukumar Vijayaraghavan (University of Colorado Denver, Anschutz Medical (ampus) for helpful advice and discussions.

Correspondence should be addressed to Nathan Schoppa, Department of Physiology and Biophysics, University of Colorado Denver, Anschutz Medical Campus, Mail Stop 8307, P.0. Box 6511, Aurora, C0 80045. E-mail: nathan.schoppa@ucdenver.edu.

DOI:10.1523/JNEUROSCI.2368-09.2009

Copyright $\odot 2009$ Society for Neuroscience $\quad$ 0270-6474/09/2913454-11\$15.00/0
2007). PG cells may be activated by multiple excitatory mechanisms at a glomerulus, including direct inputs from olfactory receptor neurons (ORNs) (Pinching and Powell, 1971; Hayar et al., 2004b; Murphy et al., 2004), and usually provide a GABAergic output into the same glomerulus at which they receive ORN inputs. Thus, PG cells could be part of a local "self-inhibitory" circuit, in which ORN inputs into a glomerulus initiate inhibition of the same glomerulus. In addition, PG cells are very small cells (5-8 $\mu \mathrm{m}$ soma diameter) with high input resistances $(\sim 1 \mathrm{G} \Omega$ ) (Puopolo and Belluzzi, 1998; Smith and Jahr, 2002), suggesting that they could be activated by weak ORN inputs. This effect could be important for preferential filtering of weak odor signals. A last point pertains to the locus of PG cell inputs with respect to mitral cell excitation. Slice studies (Carlson et al., 2000; Schoppa and Westbrook, 2001) have shown that mitral cell responses to relatively strong olfactory nerve $(\mathrm{ON})$ inputs are dominated by a $\sim 10 \mathrm{mV}$ long-lasting depolarization (LLD). LLDs are initiated within glomeruli, and, so, GABAergic inputs from PG cells in glomeruli should be well positioned to suppress LLDs.

Here, we assessed synaptic processing within bulb glomeruli, using a combination of patch-clamp recordings and calcium imaging in rat bulb slices. The study is divided into two parts. First, we followed up published studies indicating that mitral cell responses are dominated by LLDs after strong ON stimulation by examining responses across varying stimulus conditions. These experiments show for the first time that a glomerulus has two distinct response modes, either entirely active or not active, which vary in probability with ORN input level. These results form the basis for additional experiments that examined the impact of PG cells on glomerular excitation. 


\section{Materials and Methods}

General slice preparation. All experiments were conducted under protocols approved by the Animal Care and Use Committee of the University of Colorado Denver, Anschutz Medical Campus. Horizontal slices (300$400 \mu \mathrm{m})$ from rat olfactory bulbs were taken from 10- to 16-d-old Sprague Dawley rats after general halothane anesthesia and decapitation, as described previously (Schoppa et al., 1998). Bulb slices were placed on an upright Carl Zeiss Axioskop 2FS microscope with differential interference contrast optics video microscopy and a CCD camera. Cells were visualized with a $40 \times$ Carl Zeiss water-immersion objective. All experiments were done at $32-35^{\circ} \mathrm{C}$.

Electrophysiology. The base extracellular recording solution contained the following (in mM): $125 \mathrm{NaCl}, 25 \mathrm{NaHCO}_{3}, 1.25 \mathrm{NaH}_{2} \mathrm{PO}_{4}, 25$ glucose, $3 \mathrm{KCl}, 2 \mathrm{CaCl}_{2}$, and $1 \mathrm{MgCl}_{2}$, pH 7.3 (oxygenated with $95 \% \mathrm{O}_{2}, 5 \%$ $\mathrm{CO}_{2}$ ). The pipette solution for most experiments contained the following (in mM): $125 \mathrm{~K}$-gluconate, $2 \mathrm{MgCl}_{2}, 0.025 \mathrm{CaCl}_{2}, 1$ EGTA, $2 \mathrm{NaATP}, 0.5$ $\mathrm{NaGTP}$, and $10 \mathrm{HEPES}$, pH 7.3 with KOH. Mitral cell IPSCs in Figure $5 \mathrm{C}$ were recorded with a pipette solution in which $\mathrm{K}$-gluconate was replaced with $\mathrm{KCl}$.

Current and voltage signals were recorded with an Axopatch 200B amplifier (Molecular Devices), low-pass filtered at $1 \mathrm{kHz}$ using an eightpole Bessel filter, and digitized at $10 \mathrm{kHz}$. Local field potential (LFP) recordings were made with low resistance $(1-5 \mathrm{M} \Omega$ ) patch pipettes filled with extracellular solution and placed onto glomeruli. LFP signals were further bandpass filtered offline using a four-pole Butterworth filter at $0.5-20 \mathrm{~Hz}$. Loose cell-attached (LCA) recordings of action potentials were made in voltage-clamp mode with patch pipettes (5-7 $\mathrm{M} \Omega$ resistance) filled with extracellular solution. Data were acquired using AxoGraph software on a Macintosh Power Mac G5 computer and analyzed using AxoGraph and Matlab software.

ON stimulation was most commonly done by placing a patch pipette in the ON layer, $50-100 \mu \mathrm{m}$ superficial to the glomerular layer. Single stimulus pulses were applied ( $0.1 \mathrm{~ms}$ duration), with an interstimulus interval of $30 \mathrm{~s}$. During experiments investigating the behavior of LLDs at perithreshold intensities, stimulation intensity was adjusted to evoke LLDs during $20-70 \%$ of trials. For the calcium imaging experiments in Figure 6, 3 of 10 slice experiments in which PG cells were imaged were done using ORN axon bundle stimulation as a stimulation method; all granule cell imaging experiments were done using ORN bundle stimulation.

Focal puff application of $\mathrm{GABA}_{\mathrm{A}}$ receptor blockers (gabazine and bicuculline methiodide) was conducted using a picospritzer (Parker Instrumentation). Spread of drug solution was monitored with $1 \%$ phenol red in the solution. In these puffer experiments, target glomeruli were determined by imaging the apical dendrites of Alexa- 488 -filled mitral cells.

Fluorescence imaging. In experiments in which information about mitral, external tufted (ET), or PG cell anatomy was required, cells were either loaded with 100-200 $\mu \mathrm{M}$ Alexa-488 through the whole-cell patch pipette or, in some cases, loaded via single-cell electroporation (1 mM Alexa-488 in electroporating pipette) (Nevian and Helmchen, 2007). To identify pairs of mitral cells projecting to the same glomerulus for LCA recordings (see Fig. 3B), many mitral cells (12-20) were filled using single-cell electroporation, and their glomerular affiliations were examined. Alexa- 488 was excited by whole-field epi-illumination (480-510 $\mathrm{nm}$ ) using a Sutter Lambda DG-4 light source, and fluorescence signals were detected by a CCD camera (CoolSnap II HQ) under control of Slidebook imaging data acquisition software (Intelligent Imaging Innovations). Optical filters for epifluorescence included emission filters from Chroma Technology. For display purposes, image stacks were acquired, and out of focus fluorescence was removed with a Frangi-type morphological filter using Matlab software, following described methods (Sato et al., 1998).

During experiments investigating ET cell responses, we identified ET cells initially based on their location in the inner portion of the glomerular layer and their relatively large cell body size ( $\geq 15 \mu \mathrm{m}$ length). In all ET cells, we confirmed cell identity during the patch-clamp recordings based on their relatively high capacitance $(\geq 10 \mathrm{pF})$ compared with PG cells, as well as an anatomical profile that included extensive dendritic arbors and axons directed toward the mitral cell layer (see Fig. 4D). Details of methods used for identifying PG cells are provided in Results.

During calcium imaging experiments (see Fig. 6), slices were first loaded by incubation with fura- $2 \mathrm{AM}(20 \mu \mathrm{M}, 30 \mathrm{~min}$ incubation in oxygenated extracellular solution at room temperature). After loading, slices were transferred to the recording chamber, fura- 2 was excited by whole-field epi-illumination ( 380 and $340 \mathrm{~nm}$ ), and fluorescence signals were captured at a $2-4 \mathrm{~Hz}$ frame rate using Slidebook software. Changes in intracellular calcium concentration were expressed as relative $340 \mathrm{~nm} /$ $380 \mathrm{~nm}$ ratio changes $(\Delta R / R)$ measured in a region of interest. For display purposes, ratiometric fura signals after stimulation were convolved with a disk (diameter of 2 pixels) and pseudocolored.

Data analysis. For quantifying the mitral cell response magnitude in voltage-clamp recordings (see Figs. 1,2), currents evoked by ON stimulation were integrated, generally using a $0.5 \mathrm{~s}$ integration window starting just after the stimulus. This integration time window was chosen because it would capture most of the LLD response in trials in which LLDs occurred. In a minority of mitral cells, ON stimulation resulted in complex responses that included multiple LLDs in succession over a few seconds. However, the choice of the integration window duration had little bearing on our results for across-condition comparisons (see Results analyzing stimulus intensity dependence of LLD response magnitude).

Statistical significance was usually established using Student's $t$ test. Values are reported as mean $\pm \mathrm{SE}$. In experiments examining the bimodal nature of mitral cell current responses, the best fits of unimodal and bimodal Gaussian distributions to the amplitude distributions were established using unconstrained nonlinear optimization (routine in Matlab). The sum of squared errors was then calculated, and the best fit was determined using an $F$ test, accounting for the increased degrees of freedom in a bimodal fit.

\section{Results}

\section{Two excitatory response modes}

As a starting point for analyzing synaptic processing within olfactory bulb glomeruli, we examined excitatory response profiles in the bulb. Previous studies have shown that mitral cells respond to relatively strong ON stimulation with large LLD events (Carlson et al., 2000; Schoppa and Westbrook, 2001), but neuronal responses across varying levels of ORN inputs have not been closely examined. The specific issue we wanted to address was whether mitral cell responses across different $\mathrm{ON}$ stimulus intensities are graded with changing ORN input levels.

In current recordings in single mitral cells in response to ON stimulation (single shocks, $100 \mu$ s duration, 2-200 $\mu \mathrm{A}$; holding potential of $-70 \mathrm{mV}$ ) (Fig. $1 \mathrm{~A}$ ), we in fact found more complex, nongraded behavior that appeared to reflect two distinct response modes. As expected, the strongest stimulation consistently elicited large, long-lasting currents (half-width duration of $210 \pm 32 \mathrm{~ms} ; n=9$ ) that underlie LLDs (Carlson et al., 2000), but weaker stimuli resulted in either weak but nonzero responses or strong LLD responses that varied in probability depending on the stimulus strength. The bimodal behavior was most striking at moderate stimulus intensity levels (Fig. $1 A, 40 \mu \mathrm{A}$ data), in which responses went back and forth across trials between largeamplitude LLD currents and small-amplitude currents, and the derived response magnitude histograms based on current integrations (Fig. 1 B, $0.5 \mathrm{~s}$ integration window) (see Materials and Methods) had two discrete peaks (always better fitted by a sum of two Gaussian functions rather than one; $p<10^{-7} ; n=9$ mitral cells). In addition, at these perithreshold stimuli (percentage responses showing LLDs, $20-67 \%$; $n=9$ ), the LLD currents were indistinguishable in magnitude from LLDs at stimulus intensities that were 5- to 10 -fold higher [ $24 \pm 22 \%$ increase in integrated current at higher intensity stimulation for $0.5 \mathrm{~s}$ integration window, $n=8, p=0.24$ (Fig. $1 C$ ); $78 \pm 58 \%$ increase for $2 \mathrm{~s}$ inte- 
A

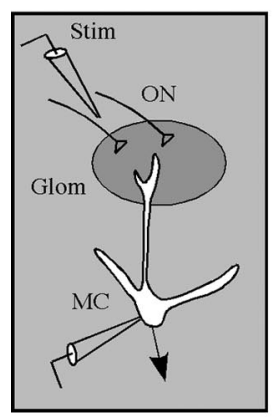

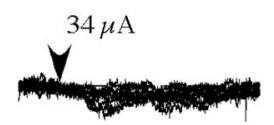

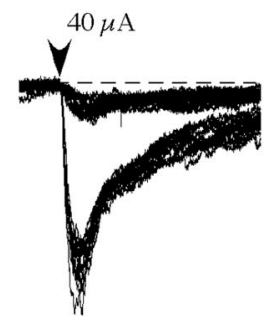

$100 \mu \mathrm{A}$

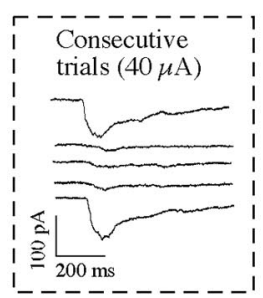

B
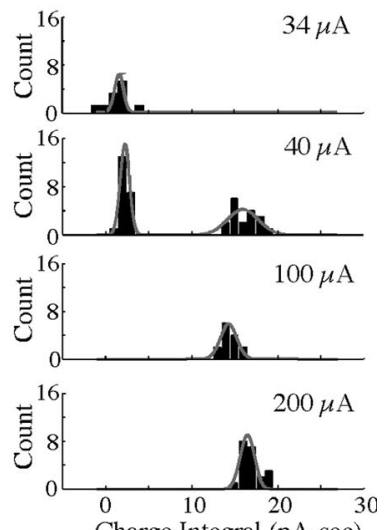

C

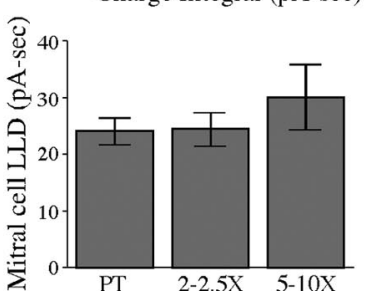

Figure 1. Bimodal synaptic responses in single mitral cells. $A$, Current responses in a single mitral cell (MC) to stimulation of the $0 \mathrm{~N}$ at various intensities (indicated; 10 traces each). Stimulation (Stim) at increasing intensity revealed two classes of current responses based on size, with the larger of the two reflecting LLDs. The probability of an LLD increased with increasing stimulus intensity. The boxed inset shows five consecutive responses at $40 \mu \mathrm{A}$. Glom, Glomerulus. $\boldsymbol{B}$, Charge integral histograms for the recording in $\boldsymbol{A}$, at varying intensities between 34 and $200 \mu \mathrm{A}$. Note the two discrete peaks for $40 \mu \mathrm{A}$ stimulation and also that the larger-amplitude peak corresponding to LLDs shifts only slightly in position for different intensities. The histogram at $40 \mu \mathrm{A}$ was well fitted by the sum of two Gaussian functions. Current integration for the determination of charge was done in a $0-500$ ms window after ON stimulation. C, Summary of the LLD charge measurements at perithreshold (PT) stimulus intensities and at higher stimulus intensities (2- to 2.5 -fold and 5 - to 10 -fold higher than perithreshold; $0.5 \mathrm{~s}$ current integration window).

gration window, $n=8, p=0.19]$. Thus, LLDs, when they happen, do not vary significantly in size with differing levels of ORN input. De Saint Jan and Westbrook (2007) reported that mitral cell synaptic responses were larger at stimulus intensities higher than those that produced what they referred to as "all-ornone" responses. However, comparisons between their study and ours are complicated by the fact that their synaptic responses were much longer lasting than the LLD responses we measured and, also, by their definition of all-or-none responsiveness (which appears to include complete response failures).

We next tested the extent to which the bimodal response behavior seen in single mitral cells extended across a glomerulus by recording LFPs at a glomerulus (Karnup et al., 2006). These signals should mainly reflect the contribution of synaptic events on dendritic processes of cells affiliated with a glomerulus. As was the case for the mitral cell current response, the glomerular LFP evoked by $\mathrm{ON}$ stimulation at moderate stimulus intensities (17-38 $\mu \mathrm{A})$ appeared to fall into two discrete groups based on size (Fig. 2A-C) (amplitude histograms always better fitted by two Gaussians functions rather than one; $p<0.03 ; n=10$ ). In addition, when we compared the glomerular LFPs with simultaneously recorded current responses in a single mitral cell affiliated with the same glomerulus (determined by including Alexa-488, $200 \mu \mathrm{M}$, in the patch electrode) (Fig. 2B), the large-amplitude glomerular LFP consistently co-occurred with the mitral cell LLD. This behavior was apparent in the clustering of data points in plots that related LFP magnitude to the mitral cell current response across trials (Fig. 2D) (seen in five of five dual recordings). These results suggest that, when any one mitral cell associated with a glomerulus undergoes an LLD event, many or all cells associated with that glomerulus also undergo an LLD. We will refer to this response as the "glomerular LLD." The LFP measured in a glomerulus reflects multiple cell types, but we will show, based on paired mitral/mitral cell recordings (see Fig. $3 A$ ) and paired mitral/external tufted cell recordings (see Fig. $4 A-C$ ), that response co-occurrences extend across specific populations of glomerular output cells. The near-perfect relationship between the glomerular LFP and the mitral cell response [which was seen previously at a qualitative level in vivo by Chaigneau et al. (2007) ] also has practical advantage for some experiments that will follow, because it means that the glomerular LFP signal can be used as a simple assay for when glomerular output neurons undergo an LLD.

Published studies have shown that LLDs dominate the synaptic response of mitral cells to strong ON stimulation and can also synchronize different mitral cells when LLDs occur (Carlson et al., 2000; Schoppa and Westbrook, 2001). Our experiments examining mitral cell and glomerular synaptic responses across different ON stimulation intensities extend on these results by providing evidence for distinct bimodal behavior across a glomerulus, in which LLDs are one of two response classes. Moreover, differing ORN input levels appear to be represented by differences in the probability that a glomerular LLD is evoked.

\section{Properties of LLD-mediated spike output for a glomerulus}

We next wondered what impact the bimodal synaptic response profile of a glomerulus had on its spike output. Given the large difference in size of the weak versus strong LLD-mediated glomerular synaptic responses (Fig. 1), one possibility is that the spike output of a glomerulus is entirely "on" or "off," with either all cells in a glomerulus spiking when there is a synaptic LLD or no cells spiking when there is not. Such a model would have functional consequences downstream in the olfactory cortex, because it would mean that all mitral cells affiliated with a glomerulus would be carrying uniform information about whether a given OR type had been activated. 
A

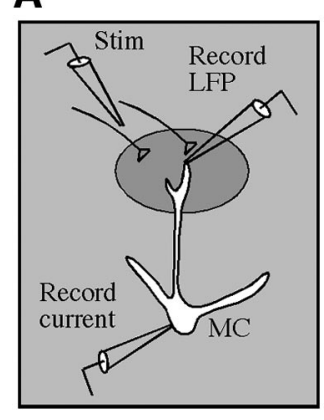

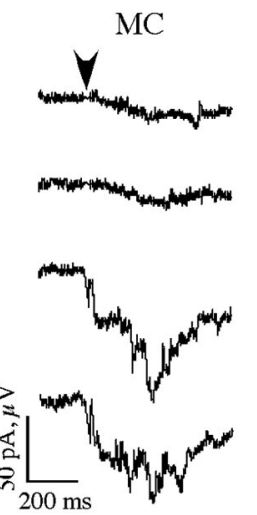

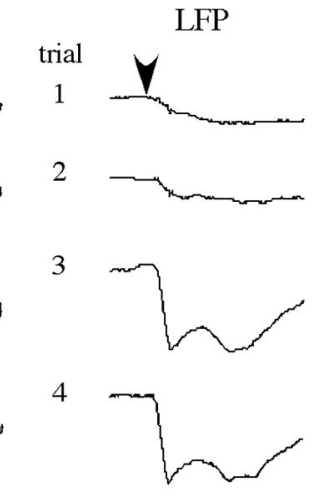

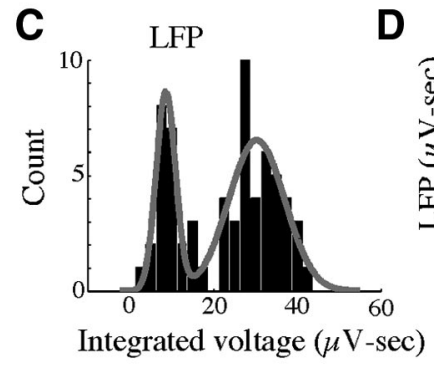

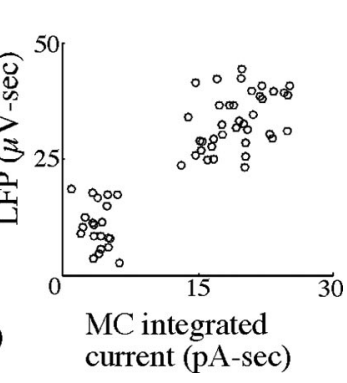

Figure 2. Evidence that a glomerulus displays bimodal synaptic responses. $A$, Simultaneous recordings of currents in a single mitral cell (MC) and the LFP signal in the glomerulus with which the mitral cell was affiliated. During the four displayed responses to ON stimulation (Stim) $(30 \mu \mathrm{A})$, the LFP signal, like the mitral cell current, displayed two discrete response types. The LFP was bandpass filtered between 0.5 and $20 \mathrm{~Hz}$. $\boldsymbol{B}$, The glomerular tuft of the test mitral cell in $\boldsymbol{A}$ (filled with Alexa 488). The glomerulus from which LFP measurements were made is demarcated by dashed ring. $C$, Histogram of response amplitude (integrated voltage) constructed from the LFP recording in $\boldsymbol{A}$. D, Plot of integrated mitral cell current versus integrated glomerular LFP for the experiment in $\boldsymbol{A}$. Each data point reflects a single trial. Note the two discrete clusters of points, indicating that large LFP responses co-occurred with LLDs in mitral cells.

We tested the on/off glomerular output model, first, using simultaneous recordings of spiking in two mitral cells affiliated with the same glomerulus, identified using electroporation of Alexa-488 (1 mu dye in the electroporating pipette). Figure 3, A and $B$, shows an example of such a pair recording, done using the LCA method, in which spike barrages (mean, $5.6 \pm 2.8$ spikes; $n=8$ cells) were evoked by ON stimulation at one intensity during some stimulus trials but not others. One prediction of the on/off model is that spiking in the two mitral cells should cooccur, that is, either both cells should spike or neither should spike. Based on four pair LCA recordings, we almost always found this to be the case (Fig. 3D) (co-occurrence rate of $93 \pm 2 \%$ ). The on/off model was further supported by another type of dual measurements, between spike activity in single mitral cells and glomerular LFPs (Fig. 3C). Here we tested for co-occurrences of mitral cell spiking and the glomerular LLD, because the on/off model requires that mitral cell spiking be perfectly associated with the glomerular LLD and that there be no "stray" spikes that happen without an LLD. Here again we found a very high co-occurrence rate of $99 \pm 1 \%(n=$ 5 pair recordings) (Fig. 3D). These results, together with results from the dual LCA recordings, indicate that mitral cells only spike when there is a glomerular LLD and that, when an LLD happens, virtually all mitral cells spike. The small errors (7 and 1\%) in the average co-occurrence rates in mitral/mitral and mitral/LFP dual recordings were always associated with single-spike responses in mitral cells.

The on/off glomerular output model makes one other prediction for the mitral cell LCA/LFP recordings, which is that the mitral cell spike rate should depend only on whether an LLD happens and otherwise be independent of input strength into a

glomerulus. To test this, we counted the number of mitral cell spikes at different stimulus intensities across a $2 \mathrm{~s}$ window after ON stimulation but excluded trials in which LLDs did not occur (Fig. 3E). This analysis showed that there was in fact no correlation between $\mathrm{ON}$ stimulation intensity and spike count (correlation coefficient, $-0.05 \pm 0.24$ across a range of stimulus intensities in which the LLD probability varied from 54 to $100 \% ; n=8$ LCA recordings), consistent with the on/ off glomerular output model. Interestingly, we did find a relationship between stimulus intensity and spike timing, with higher stimulus intensities resulting in a shorter latency to the first spike after ON stimulation (mean first spike latency, $150 \pm 27$ and $59 \pm 28 \mathrm{~ms}$ for perithreshold vs 5 - to 10 -fold higher stimulus intensities; $n=8 ; p=0.04)$. Mitral cell spike latency provides a potential means to carry information about ORN input intensity into a glomerulus (see Discussion).

\section{Conversion of graded ORN inputs to bimodal mitral cell response}

Our next objective was to determine the exact relationship between ORN inputs into a glomerulus and the glomerular postsynaptic response by recording each simultaneously. The most plausible interpretation of the changes in LLD probability with increasing ON stimulus intensity (Fig. $1 A$ ) is that there are postsynaptic mechanisms in a glomerulus that convert graded increases in ORN input levels into a glomerulus into an all-ornone glomerular LLD. However, it is also possible that the bimodal nature of the mitral cell response has a presynaptic origin. For example, ORNs could engage in electrical coupling (Zhang and Restrepo, 2003), which could promote the concerted activation of ORN axon fibers during some stimulus trials, resulting in LLDs, but not other trials.

To examine the relationship between ORN input levels and LLDs, we used recordings from a second class of excitatory neurons, external tufted (ET) cells, which surround glomeruli (Fig. 4) (for ET cell identification method, see Materials and Methods). A recent study suggested that ET cells may play an important intermediary role between ORN inputs and mitral cell activation (De Saint Jan et al., 2009). In addition, ET cell measurements provided a convenient way to assay both ORN input and glomerular LLD signals simultaneously, because both response components could be easily observed in individual ET cell records. This was apparent in mitral/ET cell pair recordings (Fig. $4 A-C$ ), in which the ET cells displayed a short-onset EPSC (delay time $=2.9 \pm 0.2 \mathrm{~ms} ; n=7$ ), indicative of monosynaptic ORNto-ET cell transmission (Hayar et al., 2004b), as well as a longlasting LLD-like current (half-width of $330 \pm 85 \mathrm{~ms} ; n=7$ ) that perfectly co-occurred with the mitral cell LLD (co-occurrence seen in six of six pairs). We considered the possibility of using mitral cell recordings for simultaneous assessment of ORN inputs and LLDs. However, we were never able to observe monosynaptic EPSCs in mitral cells indicative of ORN-to-mitral cell 
transmission (Fig. 4A, mitral cell traces) ( $n=12$ cells) (see Discussion).

The ORN input/glomerular postsynaptic response relationship was assessed by comparing the amplitude distribution of the ORN-EPSCs in ET cells with that of a delayed component of the response (200-500 ms after stimulus), corresponding to when LLDs occurred (Fig. 4D,E). In the analysis, ORN-EPSCs appeared to increase in a graded manner with increasing stimulus intensity, as reflected by a rightward shift in a single peak in the ORN-EPSC amplitude histograms (Fig. $4 E$, left) (150 $\pm 50 \%$ increase between stimuli perithreshold and suprathreshold for LLDs; $n=6$ ). At the same time, there was a corresponding change in LLD probability (Fig. $4 E$, right, see changing twopeaked histograms). Thus, it appears that there is a conversion of graded ORN inputs into a glomerulus into a bimodal mitral cell response. The comparison between ORN-EPSCs and LLDs was complicated somewhat by the fact that the ORN-EPSCs recorded in one ET cell only reflected inputs into that cell, whereas LLD generation was likely influenced by inputs across a population of cells at a glomerulus. However, the relation seen in Figure $4 E$, showing graded increases in ORN-EPSCs associated with a simple LLD probability change was observed in six of six ET cell recordings, indicating that the large majority of ET cells are receiving graded changes in ORN input levels while, at the same time, LLD probability changes.

\section{GABAergic inputs within glomeruli gate LLDs}

We next examined the impact of inhibitory synaptic processing in the glomerulus on LLDs. Given that LLDs originate in a glomerulus (Carlson et al., 2000), we hypothesized that LLDs could be strongly affected by GABAergic inputs from PG cells that surround a glomerulus. Such GABAergic inputs could have multiple functions, the most dramatic of which would involve a situation in which PG cells completely prevent generation of the LLD driven by ORN inputs. Because spiking in an entire glomerulus-specific network of mitral cells depends nearly completely on LLDs being generated (Fig. 3D), prevention of LLDs by PG cells would mean block of downstream signaling for a glomerulus.

To examine how PG cells affect LLDs, we recorded mitral cell current responses to $\mathrm{ON}$ stimulation $(10-50 \mu \mathrm{A})$ at perithreshold conditions for LLDs and tested the effect of puff application of the $\mathrm{GABA}_{\mathrm{A}}$ receptor blocker gabazine $(30 \mu \mathrm{M}$; with a patch pipette $3-4 \mu \mathrm{m}$ ) on LLD probability (Fig. $5 A$ ). We interleaved blocks of trials (4-20 trials per block) in which we preapplied gabazine for $1 \mathrm{~s}$ before $\mathrm{ON}$ stimulation with control trials in which no gabazine was applied. When drug application was onto the glomerulus with which the test mitral cell was affiliated (the "target" glomerulus) (for determination strategy, see Materials and Methods), gabazine caused a significant increase in LLD probability (Fig. $5 B)(45 \pm 13 \%$ increase; $n=4 ; p=0.039)$, as did another $\mathrm{GABA}_{\mathrm{A}}$ receptor blocker, bicuculline methiodide (BMI) (50 $\pm 5 \%$ increase; $n=9 ; p<0.0001)$. The effect of glomerular puff application was specific to the glomerulus to which the mitral cell sent its primary dendrite, because BMI application onto an adjacent glomerulus did not affect LLD probability $(3 \pm 14 \%$ decrease; $n=3$ ) (Fig. $5 B$ ). The increase in LLD probability attributable to glomerular application of $\mathrm{GABA}_{\mathrm{A}}$ receptor blockers indicated that PG cells prevented LLDs from being generated by ORN inputs during stimulus trials that resulted in LLD failures.

Besides PG cells, the bulb circuitry includes GABAergic granule cells, which synapse onto mitral cell lateral dendrites in the external plexiform layer (EPL). However, BMI $(30 \mu \mathrm{M})$, when directly puff applied in the EPL near the test mitral cell, had no effect on LLD probability ( $15 \pm 16 \%$ decrease in LLD probability; 

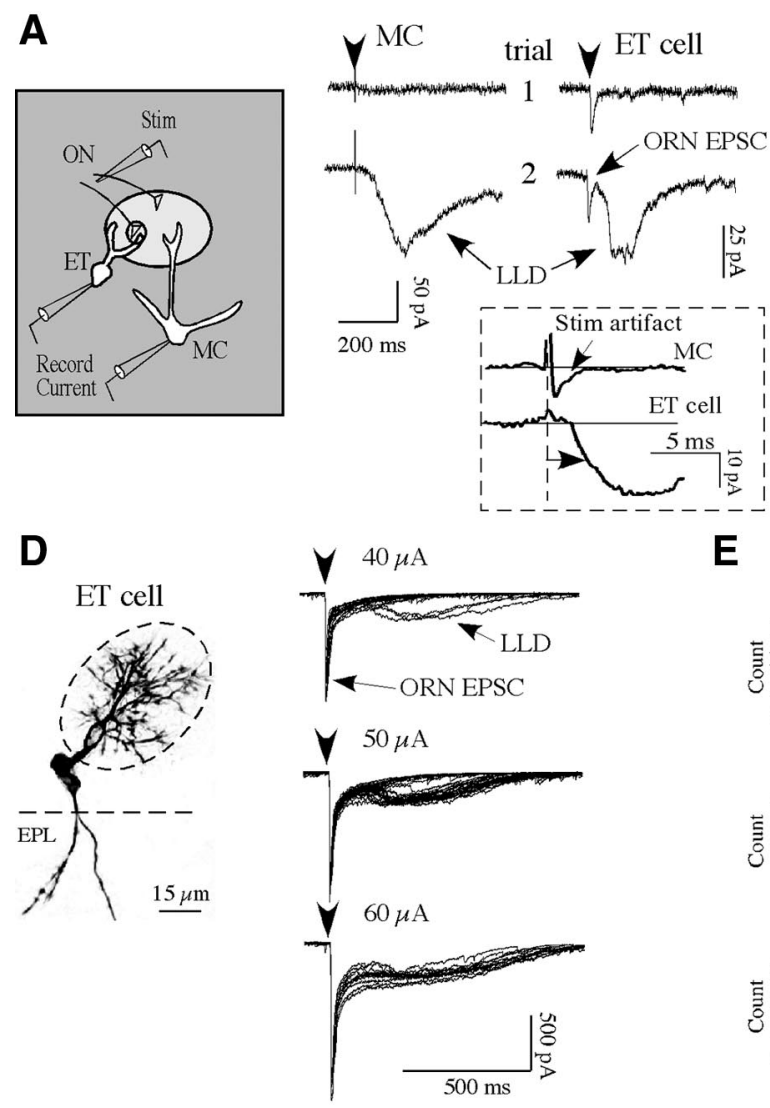

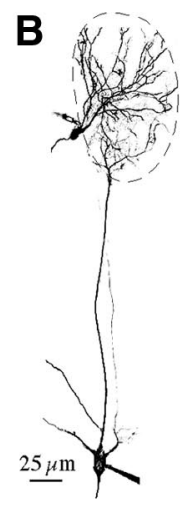

$\mathbf{E}$

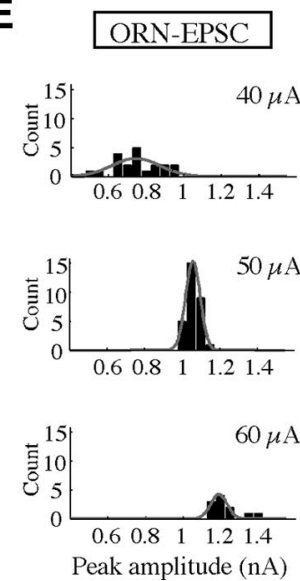

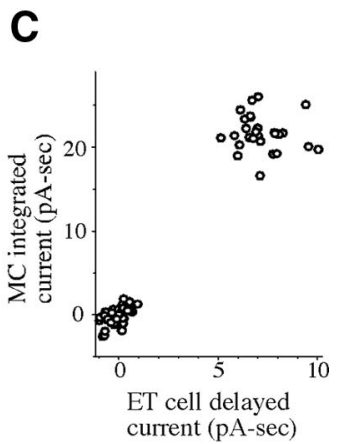

Delayed current
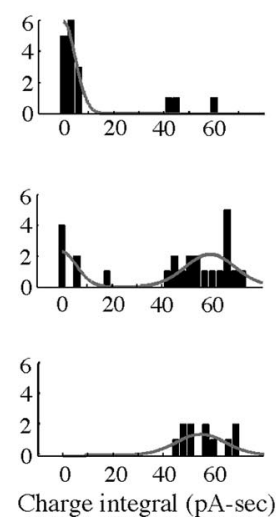

Figure 4. The glomerulus converts graded ORN inputs into a bimodal synaptic response. $A$, Simultaneous recording of mitral cell (MC) and ET cell current responses to $0 \mathrm{~N}$ stimulation (Stim) at a stimulus intensity perithreshold for LLD generation. Note that the ET, but not mitral, cell current response includes a fast EPSC closely locked to the stimulus, indicative of the ORN-EPSC. The difference in the ORN-EPSC in the mitral versus ET cell is also apparent in the expanded traces in the boxed inset. The mitral and ET cells both displayed LLD components in their responses (trial 2). B, The two cells from $\boldsymbol{A}$ filled with Alexa-488 included in the patch pipette. C, Plot relating theET and mitral cell current magnitudes (integrated charge) for the experiment in $\boldsymbol{A}$. Note the two discrete clusters of points, indicating that the large-amplitude $L L D$ events co-occurred in the two cells. For the mitral cell, current integration was done in a time window $0-500$ ms after stimulation; for the ET cell, current integration was across a delayed window 100 - $500 \mathrm{~ms}$ after stimulus to avoid the ORN-EPSC. D, Current recordings in a single ET cell at three different ON stimulus intensities. Note the moderate increase in size of the ORN-EPSC with increasing ON stimulus intensity, while at the same time there was change in the probability of the delayed LLD component of the response. $\boldsymbol{E}$, Histograms of ET cell responses at different stimulus intensities for the experiment in $\boldsymbol{D}$. The two sets of plots are for the ORN-EPSC (left, peak amplitude) and for the delayed current (right, integrated current over 200-500 ms after stimulus).

$n=6)$ (Fig. 5B), nor on the magnitude of the LLD current response ( $1 \pm 10 \%$ decrease; $n=6)$. To control for the possibility that BMI puffs in the EPL did not significantly inhibit granule cell synapses, we performed parallel measurements in which we monitored $\mathrm{GABA}_{\mathrm{A}}$ receptor-mediated IPSCs (Fig. $5 \mathrm{C}$ ) under relatively strong ON stimulus conditions $(100-200 \mu \mathrm{A})$ that have been shown previously to be effective in eliciting barrages of fast IPSCs derived mainly from granule cells (Schoppa, 2006). In these experiments, BMI puff applied in the EPL significantly decreased the peak amplitude of mitral cell IPSCs evoked by ON stimulation ( $40 \pm 6 \%$ amplitude decrease in IPSCs detected 1-2 s after stimulation; $n=6 ; p=0.01$ ). BMI puff applied on the glomerulus to which the test mitral cell sent its primary dendrites had no effect on the IPSCs ( $7 \pm 7 \%$ amplitude decrease; $n=6$ ), further confirming that the rapid IPSC barrages were mainly derived from granule cells. The failure of BMI application in the EPL to alter LLD probability, although clearly reducing GABAergic inputs from granule cells, provided good evidence that GABAergic PG cells have the exclusive role in regulating LLD probability.

\section{Evidence for a gating role for PG cells based on} calcium imaging

To further confirm the role of PG cells in gating LLDs, we turned to experiments that combined electrophysiological measure- ments of LLD responses with simultaneous activity measurements of PG cell populations affiliated with a glomerulus, using fura-2 AM dye-based calcium imaging methods ( $20 \mu \mathrm{M}$ loading concentration). The basis for these studies was provided by the puffer experiments just described (Fig. $5 A$ ): if blockade of $\mathrm{GABA}_{\mathrm{A}}$ receptors could increase the probability of the LLDs, as measured across many trials, this meant that, during trials that resulted in LLD failures under control conditions, there had to have been a population of GABAergic neurons active and shutting down LLDs. If the neurons gating the LLDs were PG cells, a comparison between LLD responses and PG cell activation should show trials in which at least some PG cells are active without an LLD. LLD responses were measured with either patch recordings of LLD currents in single mitral cells labeled with a dye (Alex-488, 200 $\mu \mathrm{M}$; to allow determination of its target glomerulus) or recordings of glomerular LFPs (Fig. 2A). To help ensure that the population of cells imaged was affiliated with a target glomerulus from which LLD measurements were made, some of these experiments ( 3 of 10) were done using a modified ON stimulation strategy in which identified ORN axon bundles were stimulated at very weak intensities $(2-5 \mu \mathrm{A})$. This method has shown previously to cause stimulation of ORNs at a single glomerulus (McGann et al., 2005). 
Figure $6 A-C$ illustrates an example of an experiment used to test a gating role for PG cells, in which we imaged a population of PG cells while simultaneously recording LLD currents in a single mitral cell affiliated with a target glomerulus. Putative PG cells selected for analysis were spherical and had very small diameters, 5-8 $\mu \mathrm{m}$ (Shepherd et al., 2004). In this experiment, we found that the number of PG cells showing calcium responses (increases in the ratio $\Delta R / R$ for $340 / 380 \mathrm{nM}$ excitation), as well as the magnitude of the calcium response, was dependent on whether an LLD occurred. However, a fraction of the PG cells clearly showed responses without an LLD. For quantifying the data across this and other experiments, we used a value of $\Delta R / R=2.5 \%$ as a threshold for whether a PG cell calcium response was associated with a PG cell spike. This value was based on parallel experiments examining the relationship between PG spiking in LCA recordings ( $n=$ 5 ) and their calcium signals in response to ON stimulation (25-50 $\mu \mathrm{A})$ (supplemental Fig. 1, available at www.jneurosci.org as supplemental material). We estimated, based on recordings in 10 slices in which we imaged $6-21$ cells per slice (128 total cells), that the number of PG cells active without an LLD was $46 \pm 6 \%$ of those active with an LLD (based on average $\Delta R / R$ measurements obtained over 5-23 trials per recording) (Fig. $6 \mathrm{D}$ ). That some of these putative PG cells activated in the absence of LLDs were indeed PG cells was confirmed by performing whole-cell recordings after the calcium imaging. In these measurements (Fig. $6 \mathrm{~B}$ ), we found that all cells tested $(n=10)$ had the high input resistance $(>700 \mathrm{M} \Omega)$ and restricted dendritic arbor expected for PG cells. Also, in three other whole-cell recordings, we found that a short depolarization ( $10 \mathrm{~ms}$ voltage pulses to $0 \mathrm{mV}$ ) elicited GABAergic self-inhibitory currents blocked by gabazine $(20 \mu \mathrm{M})$, resembling those reported previously for GABAergic PG cells (Smith and Jahr, 2002).

Dual LLD/calcium measurements were also used to confirm the lack of involvement of granule cells in gating the LLDs (Fig. 6E; all done with weak ORN bundle stimulation). Performing a similar across-trial analysis on granule cells as for PG cells under perithreshold conditions for LLD generation, we found that a selected set of granule cells that showed large calcium signals $(\Delta R / R \geq 2 \%)$ when an LLD occurred at a target glomerulus never showed a response without an LLD (31 granule cells in 6 slices; 7-16 trials per experiment). The fact that no granule cells were ever active during trials in which LLDs failed to occur meant that granule cells could not have been involved in preventing LLDs. Finding activated granule cells was expected to be somewhat complicated by the fact that the population of granule cells activated by $\mathrm{ON}$ stimulation at a particular location might be more dispersed than PG cells. However, we accounted for this issue by moving the microscope objective to visualize a relatively broad region of the granule cell layer (200-300 $\mu \mathrm{m}$ diameter area). Moreover, it should be pointed out that we had little problem identifying active granule cells in those specific trials in which LLDs did occur at a target glomerulus. In that situation, we observed at least one active granule cell in every trial.

The results of the calcium imaging experiments, together with the BMI/gabazine puff-application studies, indicate that PG cells act alone to prevent LLD generation, which, as discussed above, is an effect of strong functional interest. Clearly, PG cells, as well as granule cells, could have functions beyond just shutting down LLDs before their generation. For example, in the calcium imaging experiments, we found that many PG cells required an LLD for activation (compare two images in Fig. 6A). These PG cells could be involved in terminating an LLD once it has started or curbing the magnitude of the LLD.

\section{Intraglomerular mechanism for inhibiting LLDs}

In our final studies, we examined the targets of GABAergic inhibition from PG cells when they prevent LLDs. Previous studies have shown that ET cells can receive inputs from PG cells (Murphy et al., 2005), but the extent of PG cell input onto mitral cells is not known. Also, in our studies we were interested in the targets of PG cell inhibition under a very specific functional situation, when PG cells are actively suppressing LLDs attributable to ORN inputs. To examine the target of PG cell inhibition during LLD suppression, we assessed inhibitory responses in ET and mitral cells evoked by ON stimulation (Fig. 7), while monitoring the status of the LLD to ensure that LLDs were being suppressed. In these studies, we found that ET cells are in fact the major targets of 
A
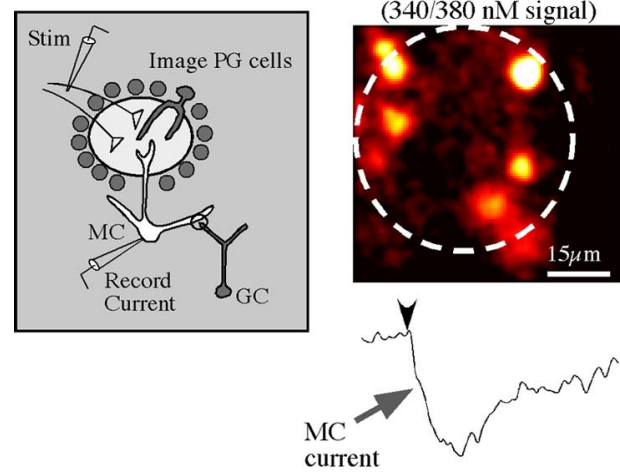

C

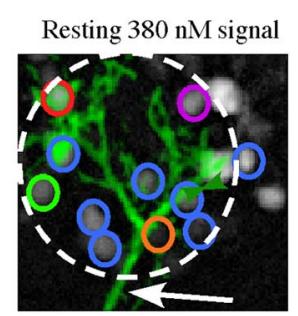

D

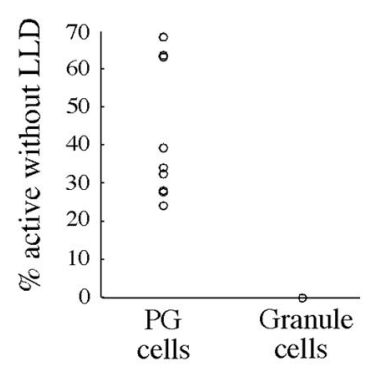

with LLD
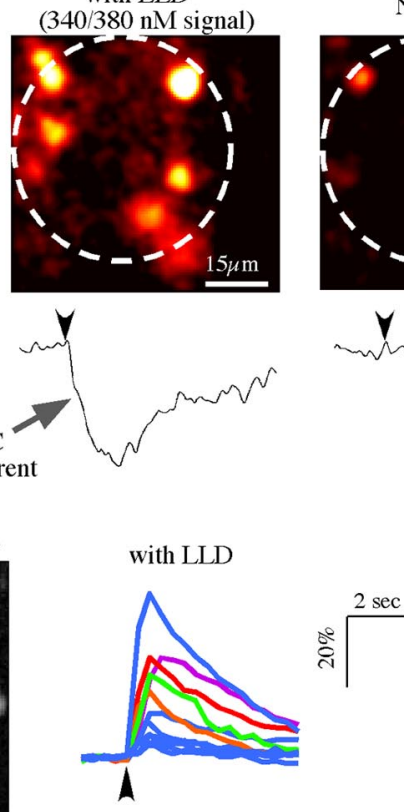

E

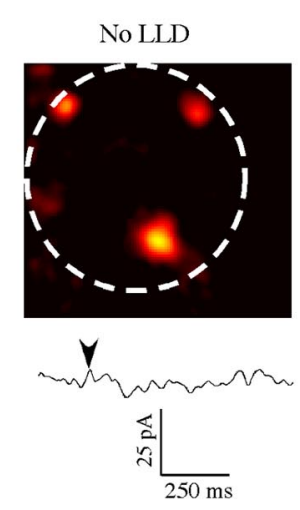

B

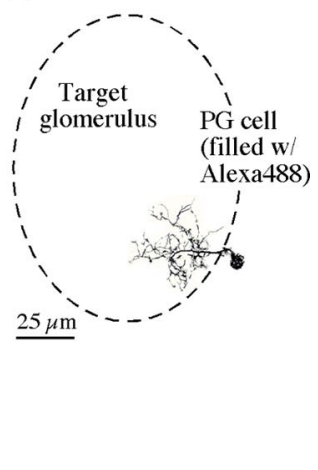

No LLD

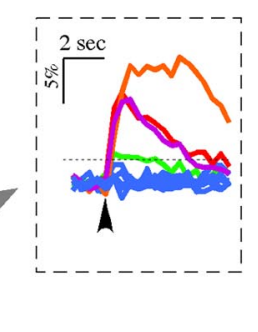
A
Granule cells with LLD

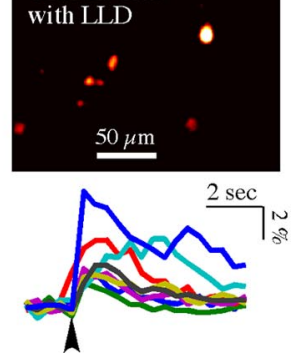

Granule cells No LLD

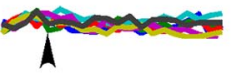

Figure 6. Evidence that $P G$ cells alone prevent LLDs, based on calcium imaging. $A$, Fura-2 calcium dye signals were imaged in PG cells surrounding a glomerulus, while LLDs were simultaneously assayed via patch-clamp recording in a mitral cell (MC) affiliated with that glomerulus. Calcium signals (top; fluorescence ratio $R$ for 340 vs $380 \mathrm{~nm}$ excitation) and mitral cell current response (bottom) in trials with (left) or without (right) an LLD. The number of active PG cells depended on the presence of an LLD, but some were clearly active without an LLD. The fura- 2 signals reflect single-frame measurements integrated $0-500 \mathrm{~ms}$ after $0 \mathrm{~N}$ stimulation (Stim). GC, Granule cell. B, An example of a PG cell determined to be active without an LLD, which was subsequently patched with an Alexa 488-containing patch electrode. Note the restricted dendritic arbor. $C$, Time courses of the changes in the fura-2 fluorescence ratio $(\Delta R / R)$ for cells in $A$. Specific cells analyzed are shown circled in the image at left of resting fura-2 signals (shown with Alexa 488-filled dendritic tuft of the test mitral cell; green). Boxed inset at right shows expanded $\Delta R / R$ time courses in trials in which LLDs failed to be evoked. Horizontal dashed line demarcates minimum $\Delta R / R$ value (2.5\%) determined to correspond to when spikes occurred in PG cells (supplemental Fig. 1, available at www.jneurosci.org as supplemental material). D, Summary of calcium measurements in PG and granule cells. Each data point reflects one experiment, showing the fraction of cells active without an LLD compared with those active with an LLD. Note the high level of activity in PG cells in the absence of LLDs and the complete lack of such activity in granule cells (no active cells in 6 experiments). $\boldsymbol{E}$, Calcium signals in granule cells (top; fluorescence ratio $R$ for 340 vs $380 \mathrm{~nm}$ excitation) in trials with (left) or without (right) an LLD. At bottom are time courses of changes in the fura- 2 fluorescence ratio ( $\Delta R / R$ ) for selected granule cells that showed clear calcium signals when an LLD occurred.

inhibition from PG cells when LLDs are being suppressed, because these cells showed robust gabazine $(20 \mu \mathrm{M})$-sensitive GABAergic currents during voltage-clamp recordings $(>50 \mathrm{pA}$ peak current amplitude at holding potential of $0 \mathrm{mV}$; half-width of $39 \pm 8 \mathrm{~ms} ; n=4)($ Fig. $7 A)$. PG cell-driven inhibition was also able to impact ET cell spike probability after ON stimulation $(64 \pm 15 \%$ increase in spike probability attributable to gabazine application; $n=3 ; p=0.030$ ) (Fig. $7 B$ ). In contrast to the strong PG cell-mediated inhibition in ET cells, we never observed an inhibitory current in mitral cells in the absence of LLDs $(n=7$ cells) (Fig. 7C). Mitral cells did show strong inhibitory responses, derived from granule cells, when they underwent LLDs (Figs. 5C, $7 C$, inset), indicating that the absence of inhibition without LLDs was not attributable to difficulty in recording inhibitory currents. Also, the complete absence of an observable inhibitory response in mitral cells was likely not attributable to dendritic filtering along mitral cell primary dendrites. Previous studies have shown that fast EPSCs reflecting "lateral" excitation between mitral cells can be routinely recorded in mitral cells (Schoppa and Westbrook, 2002; Urban and Sakmann, 2002; Pimentel and Margrie, 2008). Such EPSCs originate at mitral cell primary dendrites and, so, should be subject to similar filtering as any putative PG cellderived IPSC in the mitral cell.

Figure $7 D$ illustrates a plausible mechanism that could account for PG cell-mediated LLD suppression. This model incorporates evidence based on the study by De Saint Jan et al. (2009) that a major form of ORN-mediated excitation of mitral cells could be through an indirect path: ORN to ET cell to mitral cells. Also, as we just found, inhibition from PG cells under conditions of LLD suppression is targeted onto "intermediary" ET cells rather than mitral cells. A last important feature of the model relates to the mechanism by which PG cells are activated after ON stimulation. A previous study (Aungst et al., 2003) suggested that PG cells located at a glomerulus can be activated through a longrange interglomerular mechanism attributable to excitatory inputs from "short-axon" cells at a different, distant glomerulus. In 
our model, PG cells are activated through a local intraglomerular mechanism caused by inputs from ORNs that impinge on the same glomerulus at which PG cells are located (happening either through direct ORN inputs or an indirect path via inputs from ET cells) (Hayar et al., 2004b; Murphy et al., 2005). That PG cells can be activated through an intraglomerular mechanism would mean that, in a natural situation, PG cells would be involved in glomerular "self-inhibition," i.e., a situation in which an OR-specific signal at a glomerulus would suppress activation of the same glomerulus.

Were the PG cells that mediated suppression of LLDs in our studies activated through an intraglomerular path? The best evidence in favor of such a mechanism was provided by the subset of calcium imaging experiments described above in which we observed potent activation of PG cells at a target glomerulus in the absence of LLDs $(n=3$ glomeruli; $39 \pm 12 \%$ PG cell activation rate) using weak electrical stimulation of ORN axon bundles. Because ORN axon bundle stimulation should activate ORNs at only one glomerulus, the PG cells that suppressed the LLD at a particular glomerulus would be activated by ORNs that impinged on that same glomerulus. That there was little activation of glomeruli other than the target glomerulus with ORN bundle stimulation was also supported by our granule cell imaging experiments (Fig. 6E) (see above). In these studies, we found a perfect relationship between the occurrence of an LLD at a target glomerulus and granule cell activation across stimulus trials, with no granule cells ever being active without an LLD but some always being active when there was one. In this situation, it was very unlikely that ON stimulation inadvertently stimulated neighboring glomeruli, because, otherwise, active granule cells should have been observed during target glomerulus failures.

\section{Discussion}

In this study, we performed experiments in rat olfactory bulb slices to examine synaptic processing within glomeruli. We obtained two main novel results pertaining to the modes of excitation of a glomerulus and regulation of excitation by GABAergic neurons.

\section{Input-output relationship for a glomerulus}

First, we found that a glomerulus has mechanisms that produce an on/off response after activation of ORN inputs, which varies in probability depending on ORN input level. This behavior was observed as a bimodal distribution in response magnitude in synaptic measurements from mitral cells, with the larger response class corresponding to previously reported LLDs (Carlson et al., 2000; Schoppa and Westbrook, 2001). In addition, recordings of spike activity showed that an entire glomerulus-specific network of mitral cells either engaged in prolonged spike bursts or did not spike, depending on whether there was an LLD. Previous studies have
B ET cell (LCA)

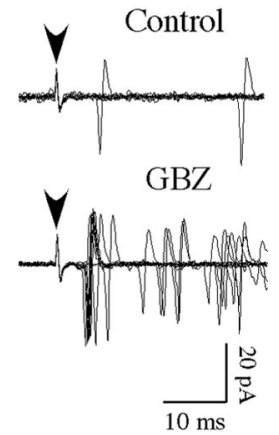

D

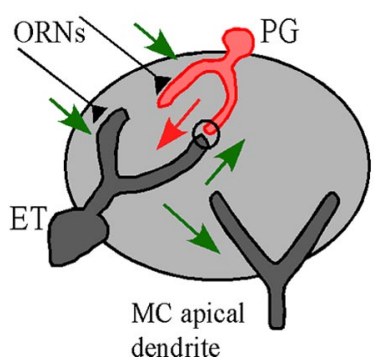

dendrite

Figure 7. ET cells are the targets of PG cell inhibition, when LLDs are being suppressed. A, GABAergic IPSC in an ET cell evoked by $0 \mathrm{~N}$ stimulation (Stim) (holding potential of $0 \mathrm{mV}$ ), under conditions in which no LLD was evoked (stimulation intensity of 10 $\mu$ A). The current was blocked by gabazine (GBZ; $20 \mu \mathrm{M})$, which revealed a remaining fast ORN-EPSC (fast downward current . The status of the LLD in this experiment was assessed from recordings in the same ET cell, by intermittently holding the

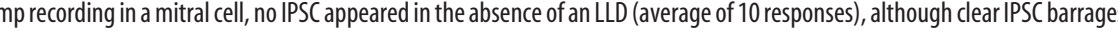
arrows in succession, ORN-to-ET-to-mitral cell). PG cells are shown to be activated by an intraglomerular mechanism, attributable to either direct ORN inputs targeted onto PG cells or an indirect path through ET cells (2 green arrows reflecting ORN-to-ET-to-PG cell).

shown that LLDs dominate mitral cell responses to strong ON stimulation and can also synchronize different mitral cells. However, our study is the first to show that, across different ON stimulus intensities, an entire glomerulus-specific network of cells has an LLD-dependent, bimodal synaptic and spike response profile.

Certainly, an important question is whether LLD-dependent glomerular on/off coding happens under natural conditions. Unfortunately, the large majority of in vivo studies have not been done in a way to allow adequate testing of an on/off scheme, although there are some studies to be considered. For example, Leveteau and MacLeod (1966) recorded slow odor-evoked glomerular field potentials and made at least a qualitative observation that they were all-or-none, similar to the bimodal responses we observed in glomerular LFP measurements (Fig. 2). A recent in vivo study by Lecoq et al. (2009) also reported LFP measurements in glomeruli, in this case, done while simultaneously measuring presynaptic calcium signals in ORNs. Their experiments provided evidence that the LFP is correlated with ORN calcium, which would appear to differ somewhat from our conclusion, based on ET cell recordings (Fig. 4), that there is a graded-to-allor-none conversion in signaling between ORNs and the glomerular response. Moreover, because EPSPs in mitral cells can be correlated to the LFPs (Chaigneau et al., 2007), the correlations of the LFPs to ORN calcium seen in the study by Lecoq et al. (2009) may extend to mitral cell postsynaptic responses. These in vivo data suggest that there could be interesting differences between 
the in vitro versus in vivo situations with respect to glomerular input-output transformations, which is a point that will need to be resolved with additional studies. The experimental methods and analyses performed in the study by Lecoq et al. (2009) differed in a number of substantial ways from our in vitro studies, for example, in how presynaptic signaling in glomeruli was monitored (ORN calcium signals vs ORN-EPSCs), and future in vitro versus in vivo comparisons will need to be done using similar methodologies.

From a mechanistic perspective, it is quite likely that the generation of an on/off glomerular output involves excitatory interactions within glomeruli. Interactions between mitral cells in a glomerulus (Schoppa and Westbrook, 2001, 2002; Urban and Sakmann, 2002; Kosaka and Kosaka 2004; Christie et al., 2005; Christie and Westbrook, 2006), either electrical or glutamatergic, should promote positive feedback excitation of the type that would lead to such behavior. However, interactions between excitatory ET cells could be at least as important given recent evidence that ET cells may have an intermediary role between ORNs and mitral cell activation (De Saint Jan et al., 2009). Indeed, in our experiments that tested for ORN-EPSCs (Fig. 4A), we obtained intriguing preliminary evidence that the indirect scheme through ET cells (ORN-to-ET-to-mitral cell) could be the dominant path for mitral cell activation, because we were never able to observe monosynaptic ORN-EPSCs indicative of direct ORN-tomitral cell synapses. It should be pointed out that this result differs somewhat from the study of De Saint Jan et al. (2009), which reported that $\mathrm{ON}$ stimulation at relatively high intensities can result in ORN-EPSCs in mitral cells. One explanation for the discrepancy is that the fast synaptic events seen in other studies reflect direct activation of mitral cell primary dendrites by the stimulating electrode, resulting in lateral excitation between mitral cells (Schoppa and Westbrook, 2002; Urban and Sakmann, 2002; Pimentel and Margrie, 2008). In our experiments, we routinely found that moderate-to-strong electrical stimulation near glomeruli resulted in directly evoked action potentials in mitral cells (data not shown), which would naturally lead to lateral excitation.

\section{Functional implications of glomerular on/off coding}

For olfactory information processing, an on/off glomerular coding scheme supported by LLDs could have a number of implications. Most importantly, such a scheme would remove much of the heterogeneity across mitral cells associated with a glomerulus and carrying information about one OR. During any one bout of input from ORNs, what we would propose to be one round of inspiration in vivo, all OR-specific mitral cells would be spiking and signaling that the OR has been activated or none of the cells would be spiking. Such a mechanism should disambiguate odor signals as they pass to the olfactory cortex.

How might more complex, second-order information about an odor be carried, for example, about odor concentration? The on/off scheme implies that odor concentration could not be coded by differences in the number of active mitral cells at a glomerulus, but there are alternate possibilities (Koulakov et al., 2007). One is based on the number of LLD-driven spikes. Our analysis of the stimulus intensity of spiking (Fig. $3 E$ ) indicated that, as long as an LLD occurs, spike count is independent of input intensity; however, because the probability of an LLD is itself dependent on input intensity (Fig. $1 \mathrm{~A}$ ), information about odor concentration could be obtained from the LLD probability. This might best be done in an integrative manner, based on how many LLDs occur across multiple rounds of ORN inputs (across multiple sniffs). Odor concentration could also be coded by the latency to first spike in mitral cells (Margrie and Schaefer, 2003), which is a mechanism supported by our finding that higher intensity $\mathrm{ON}$ stimulation reduced spike latencies. In this situation, there could be two coding mechanisms operating at the same time that signal different types of information to the cortex. Whether a specific OR is activated at all by an odor would be encoded by whether an LLD happened in its corresponding glomerulus, whereas the intensity of OR activation would be encoded by spike latency.

\section{Local inhibitory mechanisms of signal filtering}

Our second major result pertains to the regulation of LLDs, showing that a population of GABAergic PG cells can completely prevent LLD generation attributable to ORN inputs. This conclusion is based on our results showing that puff application of $\mathrm{GABA}_{\mathrm{A}}$ receptor blockers on glomeruli increased the probability of evoking the LLD. Also, calcium-imaging studies showed that PG cells could be activated without LLDs at a glomerulus, which would be required if they prevent LLDs. It appears, based on our studies (Fig. 7), that the specific way that PG cells gate LLDs is by interacting locally with ET cells.

Ours is not the first study implicating PG cells in regulation of glomerular excitation. Aungst et al. (2003) proposed that PG cells were part of a complex multicellular circuit involving short-axon cells that regulated LLD generation. However, a key difference between their mechanism and ours is related to space. In their model, the PG cell/short-axon cell circuit mediated long-range, lateral inhibitory interactions between glomeruli, whereas we propose that PG cells inhibit LLD generation through a local mechanism, wherein PG cells are activated by ORNs impinging on the same glomerulus at which they are located (Fig. 7D). Previous studies have also implicated PG cells in modifying glomerular signaling through dopamine and $\mathrm{GABA}_{\mathrm{B}}$ receptor-mediated downregulation of glutamate release from ORNs (Wachowiak and Shipley, 2006). However, such mechanisms may be mainly involved in setting tonic levels of inhibition in response to factors such as behavioral state (Pírez and Wachowiak, 2008).

A PG cell-mediated intraglomerular mechanism for inhibiting LLDs could have important functional implications for olfactory contrast enhancement (Yokoi et al., 1995). Such contrast enhancement is often attributed to lateral inhibition between OR-specific glomeruli (Mori et al., 1999; Johnson and Leon, 2007; Shepherd et al., 2007), whereby glomeruli corresponding to ORs weakly activated by an odor are filtered out by neighboring strongly activated glomeruli. However, recent evidence (Soucy et al., 2009) suggests that glomeruli may not be chemotopically well ordered in a manner suited for lateral inhibition. We propose that local intraglomerular inhibition is a better alternative for odor signal filtering (Cleland and Sethupathy, 2006). In this model, selective filtering of weak odor signals could be achieved within a local glomerular microcircuit, with a mechanism based in part on the different sizes and input resistances of PG cells versus excitatory mitral/tufted cells $[\sim 1$ G $\Omega$ for PG cells (Puopolo and Belluzzi, 1998; Smith and Jahr, 2002); $200 \mathrm{M} \Omega$ for ET cells (Hayar et al., 2004a); $30 \mathrm{M} \Omega$ for mitral cells (Chen and Shepherd, 1997)]. Weak signals would preferentially activate the highest resistance PG cells, which prevent an LLD, but stronger signals would activate mitral/tufted cells sufficiently to overcome inhibition. There is one other novel aspect of our proposed local scheme of signal filtering, which incorporates the bimodal response character of a glomerulus (discussed above). Because the output of a glomerulus can be either only entirely "on" or "off," 
PG cells would not merely alter the strength of OR-specific signals as they pass out of the bulb but would determine whether OR signals pass in their fully "on" form or do not pass at all. The result would be a simple and reduced map of OR-specific signals that can be easily interpreted by downstream cortical areas.

\section{References}

Arevian AC, Kapoor V, Urban NN (2008) Activity-dependent gating of lateral inhibition in the mouse olfactory bulb. Nat Neurosci 11:80-87.

Aungst JL, Heyward PM, Puche AC, Karnup SV, Hayar A, Szabo G, Shipley MT (2003) Centre-surround inhibition among olfactory bulb glomeruli. Nature 426:623-629.

Bozza TC, Mombaerts P (2001) Olfactory coding: revealing intrinsic representations of odors. Curr Biol 11:R687-R690.

Carlson GC, Shipley MT, Keller A (2000) Long-lasting depolarizations in mitral cells of the rat olfactory bulb. J Neurosci 20:2011-2021.

Chaigneau E, Tiret P, Lecoq J, Ducros M, Knöpfel T, Charpak S (2007) The relationship between blood flow and neuronal activity in the rodent olfactory bulb. J Neurosci 27:6452-6460.

Chen WR, Shepherd GM (1997) Membrane and synaptic properties of mitral cells in slices of rat olfactory bulb. Brain Res 745:189-196.

Christie JM, Westbrook GL (2006) Lateral excitation within the olfactory bulb. J Neurosci 26:2269-2277.

Christie JM, Bark C, Hormuzdi SG, Helbig I, Monyer H, Westbrook GL (2005) Connexin 36 mediates spike synchrony in olfactory bulb glomeruli. Neuron 46:761-772.

Cleland TA, Sethupathy P (2006) Non-topographical contrast enhancement in the olfactory bulb. BMC Neurosci 7:7.

De Saint Jan D, Westbrook GL (2007) Disynaptic amplification of metabotropic glutamate receptor 1 responses in the olfactory bulb. J Neurosci 27:132-140.

De Saint Jan D, Hirnet D, Westbrook GL, Charpak S (2009) External tufted cells drive the output of olfactory bulb glomeruli. J Neurosci 29:20432052.

Hayar A, Karnup S, Shipley MT, Ennis M (2004a) Olfactory bulb glomeruli: external tufted cells intrinsically burst at theta frequency and are entrained by patterned olfactory input. J Neurosci 24:1190-1199.

Hayar A, Karnup S, Ennis M, Shipley MT (2004b) External tufted cells: a major excitatory element that coordinates glomerular activity. J Neurosci 24:6676-6685.

Johnson BA, Leon M (2007) Chemotopic odorant coding in a mammalian olfactory system. J Comp Neurol 503:1-34.

Karnup SV, Hayar A, Shipley MT, Kurnikova MG (2006) Spontaneous field potentials in the glomeruli of the olfactory bulb: the leading role of juxtaglomerular cells. Neuroscience 142:203-221.

Kosaka T, Kosaka K (2004) Neuronal gap junctions between intraglomerular mitral/tufted cell dendrites in the mouse main olfactory bulb. Neurosci Res 49:373-378.

Koulakov A, Gelperin A, Rinberg D (2007) Olfactory coding with all-ornothing glomeruli. J Neurophysiol 98:3134-3142.

Kuffler SW (1953) Discharge patterns and functional organization of mammalian retina. J Neurophysiol 16:37-68.

Lecoq J, Tiret P, Charpak S (2009) Peripheral adaptation codes for high odor concentration in glomeruli. J Neurosci 29:3067-3072.

Leveteau J, MacLeod P (1966) Olfactory discrimination in the rabbit olfactory glomerulus. Science 153:175-176.

Margrie TW, Schaefer AT (2003) Theta oscillation coupled spike latencies yield computational vigour in a mammalian sensory system. J Physiol 546:363-374.

McGann JP, Pírez N, Gainey MA, Muratore C, Elias AS, Wachowiak M (2005) Odorant representations are modulated by intra- but not interglomerular presynaptic inhibition of olfactory sensory neurons. Neuron 48:1039-1053.
Mori K, Nagao H, Yoshihara Y (1999) The olfactory bulb: coding and processing of odor molecule information. Science 286:711-715.

Murphy GJ, Glickfeld LL, Balsen Z, Isaacson JS (2004) Sensory neuron signaling to the brain: properties of transmitter release from olfactory nerve terminals. J Neurosci 24:3023-3030.

Murphy GJ, Darcy DP, Isaacson JS (2005) Intraglomerular inhibition: signaling mechanisms of an olfactory microcircuit. Nat Neurosci 8:354-364.

Nevian T, Helmchen F (2007) Calcium indicator loading of neurons using single-cell electroporation. Pflugers Arch 454:675-688.

Panzanelli P, Fritschy JM, Yanagawa Y, Obata K, Sassoè-Pognetto M (2007) GABAergic phenotype of periglomerular cells in the rodent olfactory bulb. J Comp Neurol 502:990-1002.

Pimentel DO, Margrie TW (2008) Glutamatergic transmission and plasticity between olfactory bulb mitral cells. J Physiol 586:2107-2119.

Pinching AJ, Powell TP (1971) The neuropil of the glomeruli of the olfactory bulb. J Cell Sci 9:347-377.

Pírez N, Wachowiak M (2008) In vivo modulation of sensory input to the olfactory bulb by tonic and activity-dependent presynaptic inhibition of receptor neurons. J Neurosci 28:6360-6371.

Puopolo M, Belluzzi O (1998) Functional heterogeneity of periglomerular cells in the rat olfactory bulb. Eur J Neurosci 10:1073-1083.

Sato Y, Nakajima S, Shiraga N, Atsumi H, Yoshida S, Koller T, Gerig G, Kikinis R (1998) Three-dimensional multi-scale line filter for segmentation and visualization of curvilinear structures in medical images. Med Image Anal 2:143-168.

Schoppa NE (2006) Synchronization of olfactory bulb mitral cells by precisely timed inhibitory inputs. Neuron 49:271-283.

Schoppa NE, Westbrook GL (2001) Glomerulus-specific synchronization of mitral cells in the olfactory bulb. Neuron 31:639-651.

Schoppa NE, Westbrook GL (2002) AMPA autoreceptors drive correlated spiking in olfactory bulb glomeruli. Nat Neurosci 5:1194-1202.

Schoppa NE, Kinzie JM, Sahara Y, Segerson TP, Westbrook GL (1998) Dendrodendritic inhibition in the olfactory bulb is driven by NMDA receptors. J Neurosci 18:6790-6802.

Shepherd GM, Chen WR, Greer CA (2004) Olfactory bulb. In: The synaptic organization of the brain (Shepherd GM, ed), pp 165-216. New York: Oxford UP.

Shepherd GM, Chen WR, Willhite D, Migliore M, Greer CA (2007) The olfactory granule cell: from classical enigma to central role in olfactory processing. Brain Res Rev 55:373-382.

Smith TC, Jahr CE (2002) Self-inhibition of olfactory bulb neurons. Nat Neurosci 5:760-766.

Soucy ER, Albeanu DF, Fantana AL, Murthy VN, Meister M (2009) Precision and diversity in an odor map on the olfactory bulb. Nat Neurosci $12: 210-220$.

Urban NN (2002) Lateral inhibition in the olfactory bulb and in olfaction Physiol Behav 77:607-612.

Urban NN, Sakmann B (2002) Reciprocal intraglomerular excitation and intra- and interglomerular lateral inhibition between mouse olfactory bulb mitral cells. J Physiol 542:355-367.

Wachowiak M, Shipley MT (2006) Coding and synaptic processing of sensory information in the glomerular layer of the olfactory bulb. Semin Cell Dev Biol 17:411-423.

Yang L, Pollak GD, Resler C (1992) GABAergic circuits sharpen tuning curves and modify response properties in the mustache bat inferior colliculus. J Neurophysiol 68:1760-1774.

Yokoi M, Mori K, Nakanishi S (1995) Refinement of odor molecule tuning by dendrodendritic synaptic inhibition in the olfactory bulb. Proc Natl Acad Sci U S A 92:3371-3375.

Zhang C, Restrepo D (2003) Heterogeneous expression of connexin 36 in the olfactory epithelium and glomerular layer of the olfactory bulb. J Comp Neurol 459:426-439. 